\title{
Modeling of transient thermoelectric transport in Harman method for films and nanowires.
}

\author{
Miguel Muñoz Rojo ${ }^{1}$, Juan José Romero ${ }^{1}$, Daniel Ramos ${ }^{1}$, Diana Borca-Tasciuc ${ }^{2}$, Theodorian Borca- \\ Tasciuc $^{2}$ and Marisol Martín Gonzalez ${ }^{1}$. \\ ${ }^{1}$ Instituto de Microelectrónica de Madrid, IMM-CSIC, 8 PTM 28760 Tres Cantos, Madrid, Spain. \\ ${ }^{2}$ Rensselaer Polytechnic Institute, RPI, 110 Eighth Street, Troy, 12180, New York, USA.
}

\section{Abstract}

Harman method is a technique with great potential for rapidly scanning the figure-of-merit (ZT) of emerging nanostructured thermoelectric materials. In the AC variant of this method ZT is determined from the ratio of the electrical resistance measured across a thermoelectric material subjected to a sinusoidal current at high and low frequencies. The low frequency resistance incorporates both ohmic and Peltier responses, while at high frequencies the Peltier component vanishes. This work employs finite element modeling of the transient thermoelectric transport equations in thermoelectric thin films and nanowires to determine the frequency regime for a measurable temperature and voltage response to an applied alternating current. The lower bound for the high frequency requirement was found to depend on nanostructures' geometry (i.e. thin film thickness or nanowire diameter) and thermal properties. It is shown that reducing the thickness of the films or the diameter of the nanowires increases the lower bound for the high frequency regime, often imposing challenging conditions for the measurement of ZT. Although heat losses from the sample surface due to natural convection have little effect on measured ZT, the electrical contact resistance between the thermoelectric material and the contact electrodes can be the source for large errors. These aspects should be taken into account when performing experiments to characterize ZT using the Harman method.

Keywords: Harman technique, Thermoelectric materials, Time dependent finite element simulation, Figure of Merit.

\section{Introduction}

Thermoelectric devices produce electricity when subjected to a temperature difference and vice-versa, a cooling/heating effect when powered up. They are envisioned to play an increasingly important role in a global environment where alternative energy sources and waste heat recovery are more becoming mainstream [1]. The efficiency of thermoelectric materials is described by the figure-of-merit: $Z T=\frac{S^{2} \cdot \sigma}{k} T$, where $S, \sigma$, and $k$ are the Seebeck coefficient, the electrical conductivity and the thermal conductivity, respectively. Numerous studies carried out in the past two decades have shown the performance of thermoelectric materials is enhanced in nanostructures such as thin films, nanowires and quantum dots due to size and quantum effects $[1,2]$ and first thin film devices are now emerging on the market $[3,4]$.

One of the bottlenecks when searching for high efficiency low dimensional materials is the difficulty to rapidly assess their figure of merit. There are two different ways to determine materials' performance experimentally. The most popular is to separately measure each of the three components of ZT using different measurement techniques. However, this approach is time 
consuming and requires overcoming three different sets of challenges. In contrast, the direct measurement of $Z T$ using the Harman method requires only one experimental set-up. Hence, this method could provide a rapid way to scan for best nanostructured thermoelectric materials and could be a powerful tool for material scientists, although its application in practice is usually non-trivial as discussed next.

In the original Harman method [5], DC current is passed through a thermoelectric sample subjected to one-dimensional electrical and heat conduction along its length and insulated adiabatically. The applied current creates a temperature gradient due to opposite Peltier effects (heating vs. cooling) at the junctions between the thermoelectric sample and the two electrodes at the sample's ends. While Joule heating may occur within the sample, it does not generate a temperature difference between the electrodes because of the symmetric boundary conditions to heat transfer. At steady-state, the current is switched off, which results in an immediate drop in voltage because the ohmic component of the voltage across the sample, $V_{e}$, vanishes. However, due to the slower characteristic response of heat transport compared to the electrical transport, a temperature difference still remains across the sample, generating a Seebeck voltage, with initial value $V_{S}$. The figure of merit is then calculated from $[5,6]$ :

$$
Z T=\frac{V_{s}}{V_{e}}
$$

While this method appears simple, the measurement is non-trivial for small ZT samples or for nanostructures such as thin films or nanowires because they produce either small $V_{s}$ or very fast decaying Seebeck signals. To increase the accuracy of Seebeck voltage measurement, the technique was modified from a transient signal to electrical resistance measurements under modulated (AC) currents [7]. In the modified method, two different regimes are distinguished: a low frequency (LF or DC) regime, where the applied current produces a frequency independent steady temperature due to Peltier effect, (Fig. 1a), and a high frequency (HF) regime (Fig. 1b), where the applied voltage varies so fast that an insignificant AC temperature gradient is established. The voltage developed in the LF regime, $V_{L F}$, contains both the Seebeck and the ohmic voltage components. The voltage measured in the HF regime, $V_{H F}$, carries only the ohmic voltage. Therefore, the difference between $V_{L F}$ and $V_{H F}$ equals the thermoelectric voltage, $V_{L F}-V_{H F}=V_{S}$, and, when the same current is used to perform AC and DC measurements, the figure-of-merit can be calculated as [7]

$$
Z T=\frac{V_{L F}}{V_{H F}}-1
$$

Regardless of the method used, the key to a successful measurement is accurately determining the generated Seebeck voltage. When measuring bulk, adiabatically insulated samples having low resistance electrical contacts, the Seebeck voltage of samples with ZT 1 is of magnitude similar to the ohmic voltage and has a relatively low rate of decay which can be easily captured with an oscilloscope. However, when the same methods are applied to 
nanostructures, a small, fast decaying Seebeck voltage is typically generated, which require voltage measurement equipment with high sensitivity and high frequency response.

Because of these difficulties, the Harman methods, although have been widely used to measure the figure of merit of bulk samples [7-10], are rarely applied to thin film samples [1114] or nanowires. There are, only very few papers that measure the $Z T$ of films thinner than $6 \mu \mathrm{m}$ through these methods $[11,14]$. An added challenge to these measurements is the difficulty to ensure the validity of original conditions required by the original Harman technique (free standing, adiabatically insulated sample). For instance, measurements of thin film samples require the presence of a substrate underneath the sample. Depending on the type of substrate used, as well as the quality of contact electrodes, the frequency, voltage and gradient of temperature generated by the sample may be affected considerably. As a consequence the measured, or extrinsic, ZT can be very different from the real or intrinsic ZT of the film. Although the parasitic thermal and electrical losses have been previously studied in other works[12] and also 3D models[15] have been carried out to study the steady state response of thermoelectric devices with very little attention in its transient response, to the best of our knowledge, an in-depth study of the frequencies response during the transient Harman method for thin-film materials has not been done yet.

Therefore, the objective of this work is to determine how these non-ideal conditions affect the frequency regime. This task is undertaken for the modified Harman method, which, as described above, is more suitable to use for thin-film and nanowire characterization. First, the upper bound of the low frequency regime $\left(f_{L F}\right)$ and the lower bound of the high frequency regime $\left(f_{H F}\right)$ are determined as a function of thin film thickness or nanowire radius, thermal conductivity and power factor, as well as thermal isolation conditions (vacuum vs. natural convection) for the case of freestanding samples. In a second step, a more realistic case in which an electrical connection is attached to the top of the thin film is considered. This simplified geometry is used to study the effect of the electric connection properties and its dimensions on both the $f_{H F}$ and the obtained ZT. In all cases, the ZT value obtained from simulated data is compared with the known ZT intrinsic value of the sample to demonstrate the profound effect the non-ideal boundary conditions can have on the measurements.

\section{Numerical simulations.}

To investigate the transient thermoelectric transport in nanostructured samples, a commercially available software package COMSOL Multiphysics® was used to implement user-defined equations describing the thermoelectric effects [15]:

$$
\begin{gathered}
\rho C \frac{\partial T}{\partial t}-\vec{\nabla}\left(\left(\sigma S^{2} T+k\right) \vec{\nabla} T\right)-\vec{\nabla}(\sigma S T \vec{\nabla} V)=\sigma\left((\vec{\nabla} V)^{2}+\sigma \vec{\nabla} T \vec{\nabla} V\right) \\
\vec{\nabla}(\sigma S \vec{\nabla} T)+\vec{\nabla}(\sigma \vec{\nabla} V)=-\vec{\nabla}\left([\varepsilon] \vec{\nabla} \frac{\partial V}{\partial t}\right)
\end{gathered}
$$


In above expressions $S, \sigma$ and $k$ are the Seebeck-coefficient, the electric conductivity, and the thermal conductivity, respectively, $[\varepsilon]$ is the dielectric constant and $\rho \cdot C$ is the density multiplied by the thermal capacity of the sample. The variables are the temperature, $T$, the voltage, $V$, and the time, $t$.

These equations were rearranged to be solved using the partial differential equation (PDE) module of the finite element COMSOL ${ }^{\circledR}$ software (details are shown in the appendix). The general configuration of the sample was as illustrated in Fig.1. The Dirichlet boundary condition was applied to fix the electrically energized electrode (bottom) at room temperature. An AC square voltage waveform was applied to the other (top) electrode, letting its temperature evolve as a function of time. The mesh was selected adequately considering a minimum number of elements that gave no variations in the results when further increasing the number of nodes.

The thermoelectric material selected for these investigations is p-type $\mathrm{Bi}_{2} \mathrm{Te}_{3}$ with copper for electrodes and wires. This thermoelectric material has a high ZT and is being used in commercial room-temperature thermoelectric devices. Hence there is a great interest in exploring its properties in nanostructured forms such as thin films and nanowires [16, 17]. The temperature dependent values of the Seebeck coefficient, thermal conductivity and electrical conductivity of the sample were taken from Ref. [18].

In order to determine the effect of convective losses on the thermoelectric response, the sample of interest was modeled in air, conditions typically employed in Harman measurements on bulk samples. The size of the air-filled chamber surrounding the sample was increased until the results were size-independent. Specifically, a size of $10 \mathrm{~mm}^{3}$ was used in the modeling with side walls fixed at room temperature. Details of the equations used to model convective losses are given in appendix. Simulations with the heat convection coefficient as an input parameter have been also carried out in order to study the effects over a wide range of heat transfer coefficients.As mentioned in the introduction, the main parameters of interest in this work are the upper bound of the low frequency $(L F)$ regime $\left(f_{L F}\right)$ and the lower bound of the high frequency $(H F)$ regime $\left(f_{H F}\right)$. The former is the maximum frequency at which a steady-state temperature rise is obtained using the modified Harman technique. In practice, this frequency is of interest because it requires less time to perform the AC measurements. The latter is the minimum frequency at which no temperature gradient is generated in the sample. For these studies, this limit is defined as the condition when the temperature difference across the sample is $10 \%$ of the steady-state temperature difference, since smaller temperature gradients would produce negligible $V_{S}$ values. 


\section{Results and Discussion}

\subsection{Thermoelectric thin films}

This section illustrates the effect of sample properties and boundary conditions on the frequency regimes and the gradient of temperature generated by a free standing thermoelectric thin film. In all cases investigated here, convection from the surrounding air is considered, as it is usually the standard ambient for thermoelectric device operation. At the end of this section, a comparison is presented of the extrinsic ZT extracted for the different simulation conditions.

\subsubsection{Ideal case: Free standing cross plane simulations for intrinsic $\mathrm{Bi}_{2} \mathrm{Te}_{3}$ thin films and nanowires.}

Simulations of the voltage and temperature evolution with time for $\mathrm{p}$ type- $\mathrm{Bi}_{2} \mathrm{Te}_{3}$ films were first carried out for a simplified case, where the voltage was homogeneously applied to the top surface while the bottom surface of the film was grounded. The film had a thickness of $60 \mu \mathrm{m}$ and was freestanding in the air with the bottom face fixed at room temperature. Simulations for a film-on-substrate geometry, which are not shown here, indicated that this temperature boundary condition approximates well the transport for the studied film in the presence of a substrate with thermal conductivity higher than $1 \mathrm{~W} \cdot \mathrm{K}^{-1} \cdot \mathrm{m}^{-1}$. Fig. 2 shows the temperature and the voltage distribution along the sample thickness as a function of time for an applied pulse of $10 \mathrm{mV}$ in amplitude and 0.05 seconds in length. Due to the one-dimensional nature of the transport, the temperature and voltage are constant in the horizontal direction, so only results for the vertical cross-section of the sample are shown. The first half of the pulse period is used in the analysis, since the response for each half-period is identical, except for the polarity. The lower bound of the high frequency range $\left(f_{\mathrm{HF}}\right)$ is determined from the exponential fitting of the temperature rise (considering the time at which $\Delta \mathrm{T}=0.05 \cdot \mathrm{T}_{\max }$, where $\mathrm{T}_{\max }$ is the maximum temperature reached by the hot side and $\Delta \mathrm{T}$ is the difference of temperature).

Fig.2a shows the steady-state temperature distribution within the sample. The heating of the air in the vicinity of the upper side of the sample is clearly observed. The convective heat transfer inside the box is equivalent to a situation where the convective heat coefficient associated with natural convection from the film top and side surfaces is $10 \mathrm{~W} \cdot \mathrm{K}^{-1} \cdot \mathrm{m}^{-2}$. It shall be mentioned that results are nearly independent of this natural convection factor in a broad range of values up to about $10,000 \mathrm{~W} \cdot \mathrm{K}^{-1} \cdot \mathrm{m}^{-2}$. Moreover, when considering the vacuum condition, the results are not significantly modified confirming the small effect of natural convection for these films. Fig.2b shows the temperature at the upper side of the sample as a function of time. The temperature increases nearly exponentially with the time to steady-state is reached. Also shown in this figure is the voltage evolution as a function of time. An instantaneous increase of the voltage is produced when the pulse is applied (corresponding to the applied voltage $\mathrm{V}_{\mathrm{e}}$ ). The voltage then increases slowly due to the Seebeck component which develops a temperature difference across the sample increases. From this figure, it can be seen 
that an electric pulse with duration of less than $10 \mathrm{~ms}$ will not be sufficiently long for the temperature to reach steady state. For instance, when the electric pulse is less than $0.1 \mathrm{~ms}$ the temperature increase is lower than $1.6 \mathrm{~K}$ and the estimated Seebeck voltage is below $0.36 \mathrm{mV}$ instead of the $7.12 \mathrm{mV}$ observed in the steady state. Two time constants are defined based on this type of analysis: the one at which the temperature reaches its maximum value (for this example, approximately around $10 \mathrm{~ms}$ ), and the one at which the temperature increase is a tenth of the maximum temperature increase (approximately around $0.1 \mathrm{~ms}$ here). These values are then used to find $f_{\mathrm{HF}}$ and $f_{\mathrm{LF}}$ that will give the frequency requirements of the applied voltage for the steadystate Seebeck voltage to be accurately measured or absent, respectively.

Following this procedure, the low and high frequency regimes were determined for different thicknesses of the $\mathrm{Bi}_{2} \mathrm{Te}_{3}$ films, whose aspect ratio (area to thickness ratio) was approximately 100 (to maintain the 2-dimensional character of the geometry). The maximum temperature gradient generated in these samples was around $34 \mathrm{~K}$ for the $10 \mathrm{mV}$ applied pulse voltage. It must be noted that increasing the pulse amplitude produces an increase in the temperature difference; however the values of the low and high frequencies remain nearly unaltered.

Fig.3a and Fig.3b shows the values of the $f_{L F}$ and $f_{H F}$ and the values of the extrinsic ZT and difference of temperature reached as a function of film thickness. The data shows a significant increase of the two bounds of frequency with the reduction in the sample thickness. This indicates that the modified Harman technique becomes difficult to use for sample thicknesses under $5 \mu \mathrm{m}$, due to the high $f_{H F}$ required, over $10^{5} \mathrm{~Hz}$. Moreover, the effective ZT (from Eq. 2) is modified slightly with the thickness of the film because the temperature difference reached across sample increases for thicker films, and $S, \sigma$ and $k$ are temperature dependent so they change. However, in all cases, the simulated ZT values correspond to the theoretical intrinsic ones predicted based on properties estimated at averaged temperature. Finally, although there are not many reports of Harman measurements of $\mathrm{Bi}_{2} \mathrm{Te}_{3}$ films, the temporal evolution of voltage reported for a $\mathrm{Bi}_{2} \mathrm{Te}_{3} / \mathrm{Sb}_{2} \mathrm{Te}_{3}$ thin film superlattice of $5.4 \mu \mathrm{m}$ thickness [11] presented a similar lower bound of approximately $100 \mathrm{kHz}$ for the high frequency regime, as predicted here.

The lower bound of the high frequency regime can also be estimated from a lumped heat capacity model for the film while assuming that heat transfer occurs by conduction across the film thickness and convection to the ambient. This model leads to a simple analytical expression,

$$
f_{H F \text { theory }}=\frac{1}{R_{t h} \cdot C}=\frac{k}{\rho \cdot c \cdot\left(\text { thickness }^{2}\right)} \cdot\left(1+\frac{h \cdot(\text { thickness })}{k}\right)
$$

where $R_{t h}$ is the sample thermal resistance, $C$ is the thermal capacity, $\rho$ is the density, c the specific heat and $\mathrm{h}$ the convective coefficient. The term $\frac{h \cdot(\text { thickness })}{k}$ in the second part of Eq. 5 is much smaller than 1 , even for $h$ values of $10,000 \mathrm{~W} \cdot \mathrm{K}^{-1} \cdot \mathrm{m}^{-2}$, revealing little influence of 
convection on the high frequency regime of films. Fig.4 shows a comparison of the high frequency values obtained from Eq. 5 (red line) and the results of numerical simulations (black dots). As can be seen, there is a good agreement between both results.

The effect of the material properties on $f_{H F}$ was assessed next. The analysis was carried out on a $60 \mu \mathrm{m}$ thickness sample while the thermal conductivity and the power factor (the product between electrical conductivity and the squared Seebeck coefficient) was varied. The goal was to understand how variations of material properties alter the lower bound of the high frequency regime. Fig.5 shows $f_{H F}$ as a function of the power factor and the thermal conductivity. In Fig.5, the power factor is varied by changing the Seebeck coefficient without changing the electrical conductivity, as well as varying electrical conductivity while the Seebeck coefficient was kept constant.

From this figure, it can be seen that $f_{H F}$ depends strongly on the thermal conductivity, while it has not a noticeable dependence on the power factor. This fact can be understood taking into account the heat transfer in the sample. If the thermal conductivity of the sample is low, it takes longer to reach steady state and the frequency is low. Above a thermal conductivity of around $1 \mathrm{~W} \cdot \mathrm{K}^{-1} \cdot \mathrm{m}^{-1}$, establishing a steady-state temperature rise across a $60 \mu \mathrm{m}$ thick film, requires a lower bound for $f_{H F}$ at $0.5 \mathrm{kHz}$. Since the heat transfer depends on both thermal properties and material thickness, similar effect is obtained when the sample thickness is reduced. For example, the effect of the thermal conductivity of samples of different thicknesses and an aspect ratio of at least 100 times is shown in Fig.6. This figure illustrates the increase in $f_{H F}$ with the thermal conductivity of the sample and its inverse dependence on the material thickness, which was also observed in Fig 3a. Finally, all these results agree well with the theoretical values obtained from Eq. (5).

A similar study was performed on p-type $\mathrm{Bi}_{2} \mathrm{Te}_{3}$ nanowires $(\mathrm{NW})$ with different diameters. As before, the voltage was applied homogeneously at the top and bottom of the nanowire. The $\mathrm{Bi}_{2} \mathrm{Te}_{3}$ wire was oriented with the c-axis parallel to the NW and the same temperature dependent properties [18] were used to be consistent with previous analysis, and not considering any confinement effects since evaluating the change in thermoelectric transport properties as a function of wire diameter is outside the scope of this paper (note that size effects can usually be taken into account by replacing the bulk value of thermal conductivity with an effective value). As in the thin film case, the ground electrode was set at room temperature while the hot side of the nanowire evolved freely. The wire was freestanding and set in air at room temperature, where convection effects were considered on all its sides. The length of the nanowire was $20 \mu \mathrm{m}$ whereas the nanowire radius ranged from $50 \mathrm{~nm}$ to $250 \mathrm{~nm}$. The voltage applied to the nanowire was $10 \mathrm{mV}$, which generated temperature differences lower than one Kelvin.

Fig.7, shows the effect of the reduction in the nanowire radius on the $f_{H F}$ and $f_{L F}$. The frequency increases dramatically for 1D structures as compared with thin films, especially when their diameter is in the range of $100 \mathrm{~nm}$ or less. For a given diameter the HF and LF frequency 
limits are about two orders of magnitude apart. As an example, in order to avoid the thermoelectrical response of a freestanding nanowire of $100 \mathrm{~nm}$ radius, $f_{H F}$ should be higher than $10 \mathrm{MHz}$. The high frequencies observed in this case are due to the low time constant associated with the nanostructure, which is able to rapidly respond to a change in temperature to the applied heat. As a consequence, individual one-dimensional structures, like nanowires, are challenging to measure with the original or modified Harman method.

\subsubsection{The effect of electrical contacts}

In order to analyze how electrical and thermal contacts affect the lower bound of the high frequency regime, simulations of a thin film $\mathrm{Bi}_{2} \mathrm{Te}_{3}$ sample sandwiched between electrodes that have different transport properties were carried out. Moreover, to approach the reality, a contact wire was connected to the top electrode. Joule, Thomson and thermoelectric effects were considered for the film, the wire and the electrodes. The external face of the bottom electrode was fixed at room temperature while the temperature of the top electrode and the wire were let to evolve freely.

These simulations were first carried out on a $60 \mu \mathrm{m}$ thick p-type $\mathrm{Bi}_{2} \mathrm{Te}_{3}$ film with an area of $600 \times 600 \mu \mathrm{m}^{2}$ (width/length ten times bigger than the thickness to approach to the $2 \mathrm{D}$ situation). That is a typical film produced by electrodeposition [19] although this work can be applied to other thicknesses and fabrication techniques. The electrodes have the same area as the Bi2Te3 film and a thickness of $20 \mu \mathrm{m}$, because in soldering processes the thickness of the contact metals is often increased to this range. The contact wire was made of copper and had a diameter of 100 $\mu \mathrm{m}$ with a thermal conductivity of $400 \mathrm{~W} \cdot \mathrm{K}^{-1} \cdot \mathrm{m}^{-1}$, an electrical conductivity of $6 \cdot 10^{7} \mathrm{~S} / \mathrm{m}$ and a Seebeck coefficient of $1.7 \mu \mathrm{V} / \mathrm{K}$ [20]. To simulate the effect of a contact resistance (that depends on the quality of the contact between the film and electrode) the electrical conductivity of the film electrode was varied by around 5 orders of magnitude with copper as the higher limit. Meanwhile, the electrode thermal conductivity and specific heat were assumed to be similar to copper. The simulations recorded the steady-state temperature and voltage for each case. Fig. 8 shows the results in an electric pulse of $10 \mathrm{mV}$ and a length of 0.05 seconds.

Fig.8a shows that high electrical conductivity electrodes, i.e. low electrical contact resistances, do not degrade the temperature gradient established by the Peltier effects and the Seebeck voltage until it plateaus after about $10^{8} \mathrm{~S} / \mathrm{m}$. This corresponds to an equivalent contact resistivity of around $5 \cdot 10^{-7} \Omega \cdot \mathrm{m}$, roughly an order of magnitude smaller than the thermoelectric film resistivity. The reduction of $f_{H F}$ in Fig.8b in comparison to $f_{H F}$ in Fig.3 is due mainly to the heat dissipation along the external wire and heat capacity of the electrodes. While the $f_{H F}$ in Fig.8b will give the limit for which steady-state is achieved in the sample, performing measurements in this frequency will not result in accurate measurements if the contact resistance is high. As seen from Fig.8b the figure of merit (ZT) is profoundly affected by contact resistance and becomes impossible to measure if electrode electrical conductivity is less than $10^{8} \mathrm{~S} / \mathrm{m}$. 
Moreover, although a low contact resistivity improves the amplitude of the Seebeck and temperature signals, the contact wire also plays a major role. For instance, the steady-state temperature of the $60 \mu \mathrm{m}$ thick $\mathrm{Bi}_{2} \mathrm{Te}_{3}$ sample without the influence of any contacts was determined to be around 34 degrees and the total voltage around $17.4 \mathrm{mV}$ (Fig.2). In contrast, even for a contact resistivity below $5 \cdot 10^{-8} \Omega / m^{2}$ the maximum temperature difference reached is about 26.8 degrees while the total voltage is $15.8 \mathrm{mV}$. This corresponds to an extrinsic ZT for the film and electrode assembly of 0.58 , a reduction that will be discussed further in this section.

To study the effect of the contact wire radius, the simulations were carried out on a $\mathrm{Bi}_{2} \mathrm{Te}_{3}$ film of $60 \mu \mathrm{m}$ thickness sandwiched between $20 \mu \mathrm{m}$ copper electrodes with thermal conductivity of $400 \mathrm{~W} \cdot \mathrm{K}^{-1} \cdot \mathrm{m}^{-1}$, an electrical conductivity of $6 \cdot 10^{7} \mathrm{~S} / \mathrm{m}$ and a Seebeck coefficient of $1.7 \mu \mathrm{V} / \mathrm{K}$. In all simulations the wire was attached to the top electrode and had a length of $1 \mathrm{~mm}$. The voltage applied to the bottom electrode which was fixed at room temperature. The wire was grounded at its free end, while its temperature and that of the top electrode were let evolve freely.

Fig.9a shows the temperature distribution in the sample with a wire diameter of $100 \mu \mathrm{m}$, which illustrates the strong fin effect of the wire. Fig.9b and Fig.9c shows $f_{H F}$ and temperature difference $(\Delta \mathrm{T})$ and total voltage and ZT obtained in the film as a function of the wire diameter, respectively. Frequency, temperature difference, total voltage and the figure of merit increase when reducing the wire diameter approaching the case without wire. However, at the practical dimensions (wire radius of $50 \mu \mathrm{m}$ or larger, which can be easily attached manually) the temperature difference of around $26 \mathrm{~K}$ is still around 8 degrees smaller than the ideal case, and as a result the extrinsic ZT is around 0.56 instead of 0.74 expected. Contact wires with large diameters could result in massive heat loss and order of magnitude reduction in the measured temperature difference, resulting in an artificially low extrinsic ZT. These aspects are relevant not only to Harman measurement, but to thin film device design as well. Moreover, given these discrepancies, the analytical Eq. 5 will not work well in the presence of the electrodes and contact wires. Therefore, a revised equation to take into account these effects must be considered. In ref. [12], the wire thermal resistance was expressed as,

$$
R_{f i n}=\frac{\tanh \left(\sqrt{\frac{l^{2} \cdot h_{f i n} \cdot p_{f i n}}{k_{f i n} \cdot A_{f i n}}}\right)}{\sqrt{h_{f i n} \cdot p_{f i n} \cdot k_{f i n} \cdot A_{f i n}}}
$$

where "fin" stands for our wire and $p, A, k, l$ and $h$ are the perimeter, cross section area, thermal conductivity, length and convection on the lateral side of the wire, respectively.

Therefore, after considering the additional heat capacity of wire and electrode and the thermal dissipation along the wire the revised lumped heat capacity model yields the new frequency prediction that can be expressed as, 


$$
f_{H F}=\left(\frac{1}{R_{\text {fin }}}+k_{\text {film }} \cdot \frac{A_{\text {film }}}{\text { thickness }}+h \cdot\left(A_{\text {film }}-A_{\text {fin }}\right)\right) \cdot \frac{1}{C_{\text {total }}}(7)
$$

where "film" stands for the thermoelectric film and $C_{\text {total }}$ is the total heat capacity of the system calculated as, $C_{\text {total }}=c_{\text {fin }} \cdot \rho_{\text {fin }} \cdot V_{\text {fin }}+c_{\text {elect }} \cdot \rho_{\text {elect }} \cdot V_{\text {elect }}+c_{\text {film }} \cdot \rho_{\text {film }} \cdot V_{\text {film }}$, where $c$ is the specific heat, $\rho$ is the density and $V$ is volume. The electrode thermal resistance, $R_{\text {elec }}$ is much smaller $R_{\text {fin }}$ so its contribution doesn't need to be included in Eq.7.

Figure 10 shows a comparison of the $f_{H F}$ obtained from simulated data and Eq. 7. The wire in the simulation has a length of $1 \mathrm{~mm}$, while for Eq. 7 different wire lengths have been considered. As can be seen, in all cases a clear increase in the frequency is observed for reduced wire radius, but there is a noticeable difference in the frequency values and tendency between the simulation and the values obtained from Eq. 7 to $1 \mathrm{~mm}$-length wire, while longer wires show a tendency which resembles that of the simulated data, but with lower values. This could indicate that the 1 dimensional model given by Eq. 7 is not reproducing with high accuracy the $f_{H F}$ results obtained by three-dimensional simulation, although it can be used to obtain an order of magnitude estimation of these values. Finally, it was observed that for large diameter wires the $f_{H F}$ is mainly estimated by $\frac{1}{C_{\text {total }} R_{\text {fin }}}$, while as the diameter of the wire reduces $f_{H F}$ tends to Eq.5, as expected.

When considering just the effect of electrodes (no contact wires,), $f_{H F}$ increased to around $755 \mathrm{~Hz}$ and extrinsic ZT to 0.74 , equivalent to $\mathrm{ZT}$ determined under atmospheric conditions without electrodes. The high frequency determined for such electrode thickness without the wire is practically the same to the one determined without electrodes. Nevertheless, the reduction of the thickness of the electrodes results in approaching of the high frequency value corresponding to the one without layer electrodes $(765 \mathrm{~Hz})$.

The lower bound of the high frequency regime and the simulated values of ZT was compared for all cases discussed above, i.e. films with and without contact resistance and wire electrodes in vacuum or atmospheric conditions. The results are shown in Fig.11, where both $f_{H F}$ and ZT are plotted for each case. The frequency limit obtained under vacuum conditions was similar to the ones obtained under atmospheric conditions, regardless of the presence of wires and electrodes. On the other hand, when electrical connections ( $50 \mu \mathrm{m}$ diameter and $1 \mathrm{~mm}$ length wire) are taken into account a clear reduction of both $f_{H F}$ and $\mathrm{ZT}$ is obtained from the simulations, indicating that the thermal dissipation occurring on the wires greatly affects the Harman measurement. The ZT values obtained are reduced by nearly a $30 \%$ with respect to the intrinsic value of the material, making the determination of the figure-of-merit very inaccurate.

Therefore, to perform an accurate and direct measurement of the figure of merit very small wire diameters below $10 \mu \mathrm{m}$ shall be used as clearly seen in Fig.9, which involve a reduction of ZT of about $10 \%$. 


\section{Conclusions.}

In summary, we have studied the time dependent evolution of the temperature gradient generated by the thermoelectric effect in thin film and nanowire samples under the application of an electrical voltage. To the best of our knowledge, this is the first time that a 3D time dependent temperature model has been developed for the AC Harman method in order to determine the low frequency and high frequency associated with the measurement. Of particular interest was determining $f_{H F}$, i.e. the frequency at which thermoelectric effects are negligible.

In the case of thin film measurements in cross-plane direction, it was observed an increase of $f_{\mathrm{HF}}$ when reducing the thickness of the film. Particularly, for thicknesses under $5 \mu \mathrm{m}$ the expected $f_{H F}$ is over $100 \mathrm{kHz}$. In these cases, the Harman method requires of special electronic devices which are able to detect such small and fast signals, while reducing antenna effects.

Moreover, in this work has been observed that the Harman method is strongly dependent on the sample thermal conductivity. Indeed, evaluating $f_{\mathrm{HF}}$ for different material properties, it was observed that it hardly depends on the Seebeck coefficient or electrical conductivity, while it depends strongly on the thermal conductivity of the sample. The smaller the thermal conductivity, the lower the $f_{\mathrm{HF}}$, and then the easier the application of the modified Harman method. The dependence of $f_{\mathrm{HF}}$ with thickness, thermal conductivity and heat capacity was predicted well by a simple analytical model for the time constant of the film. However, the analytical model over-predicts $f_{H F}$ when the electrode wires play a role in heat transfer.

The values of the figure of merit, ZT, measured by the AC Harman technique have been calculated from the model under vacuum and atmospheric conditions. It was found that natural convection has negligible effects on simulated ZT. However, the heat loss associated with the contact wire and the contact resistance can profoundly affect ZT results. For example, if electrical conductivity associated with the contact is of order $10^{7} \mathrm{~S} / \mathrm{m}$, measured ZT is negligible. ZT approaches expected values, when the conductivity increases to above $10^{8} \mathrm{~S} / \mathrm{m}$. However, even under these conditions, measured ZT can be significantly lower if the diameter of the contact wire is larger than $50 \mu \mathrm{m}$. For example, a radius as small as $50 \mu \mathrm{m}$ can result in ZT errors of $30 \%$. All these findings are important to practical implementation of the modified Harman technique as well as the design of thermoelectric thin film devices.

Finally, from simulations of the AC Harman technique applied to nanowires, it was observed a strong dependence of $f_{\mathrm{HF}}$ on nanowire diameter resulting in very high frequencies, $\left(f_{H F}>\right.$ $10^{6} \mathrm{~Hz}$ for diameters below $250 \mathrm{~nm}$ ). 


\section{Acknowledgements}

M.M.G. acknowledges support from ERC StG NanoTEC 240497 and MINECO for PHOMENTA project. D.A.B.T. acknowledges support from U.S. NSF IRES-1028071. TBT gratefully acknowledges funding from the U.S. Department of Energy, Office of Science, Office of Basic Energy Sciences through the S3TEC Energy Frontiers Research Center at MIT under Award No. DE-SC0001299. M.M.R. acknowledges CSIC for their JAE pre-doctoral fellowship. M.M.R. likes to thank J. Oxaal, M. Hughes and S. Moran for useful discussions on the challenges associated with applying Harman method to thermoelectric characterization of thin films and nanowires. T. Borca-Tasciuc acknowledges partial support from Rensselaer Polytechnic Institute and from the US Department of Energy, Office of Science, and Office of Basic Energy Sciences through the S3TEC Energy Frontiers Research Center at MIT under Award Number DE-SC0001299. 


\section{APPENDIX: MODEL EQUATIONS.}

The equations introduced to COMSOL $®$ Multiphysics software are given by Antonova et al.[15] The thermoelectric equations of the heat flow and the continuity of electric charge are coupled to obtain the thermoelectric constitutive equations,

Constitutive equations $\left\{\begin{array}{l}\mathbf{q}=[\Pi] \cdot \mathbf{J}-[\lambda] \Delta \mathrm{T} \\ \mathbf{J}=[\sigma] \cdot(\mathbf{E}-[\alpha] \cdot \nabla \mathrm{T}) \\ \mathbf{D}=[\varepsilon] \cdot \mathbf{E}\end{array}\right.$
Field equations

where

$$
\begin{gathered}
\rho=\text { density, } \mathrm{kg} / \mathrm{m}^{3} \\
c=\text { specific heat capacity, } \mathrm{J} /(\mathrm{kg} \mathrm{K}) \\
T=\text { absolute temperature, } \mathrm{K} \\
\dot{q}=\text { heat generation rate per unit volume, } \mathrm{W} / \mathrm{m}^{3} \\
\mathbf{q}=\text { heat flux vector, } \mathrm{W} / \mathrm{m}^{2} \\
\mathbf{J}=\text { electric current density vector }, \mathrm{A} / \mathrm{m}^{2} \\
\mathbf{E}=\text { electric field intensity vector, } \mathrm{V} / \mathrm{m} \\
\mathbf{D}=\text { electric flux density vector, } \mathrm{C} / \mathrm{m}^{2} \\
{[\lambda]=\text { thermal conductivity matrix, } \mathrm{W} / \mathrm{Km}} \\
{[\sigma]=\text { electrical conductivity matrix, } \mathrm{S} / \mathrm{m}} \\
{[\alpha]=\text { Seebeck coefficient matrix, } \mathrm{V} / \mathrm{K}} \\
{[\Pi]=T[\alpha]=\text { Peltier coefficient matrix, } \mathrm{V}} \\
{[\varepsilon]=\text { dielectric permittivity matrix }, \mathrm{F} / \mathrm{m}}
\end{gathered}
$$

This equation was rewritten in order to fit with the partial differential equation module of COMSOL®,

$$
\begin{array}{r}
\rho c \frac{\partial T}{\partial t}-\vec{\nabla}\left(\left(\sigma \alpha^{2} T+\lambda\right) \vec{\nabla} T\right)-\vec{\nabla}(\sigma \alpha T \vec{\nabla} V)=\sigma\left((\vec{\nabla} V)^{2}+\sigma \vec{\nabla} T \vec{\nabla} V\right) \\
\vec{\nabla}(\sigma \alpha \vec{\nabla} T)+\vec{\nabla}(\sigma \vec{\nabla} V)=-\vec{\nabla}\left([\varepsilon] \vec{\nabla} \frac{\partial V}{\partial t}\right)
\end{array}
$$

The PDE equations in COMSOL $®$ are defined as,

$$
\begin{array}{cc}
k \frac{\partial^{2} u}{\partial t^{2}}+d \frac{\partial u}{\partial t}+\nabla \cdot(-k \nabla u-\alpha u+\gamma)+\beta \cdot \nabla u+a u=f & \text { in } \Omega \\
n \cdot(-k \nabla u-\alpha u+\gamma)+q u=g-h^{T} \mu & \text { on } \partial \Omega
\end{array}
$$




$$
h u=r \quad \text { on } \partial \Omega
$$

where $k, d, \alpha, \gamma, \beta, a, f, g, h^{T}, r$ are the coefficient of the differential equation and boundary coefficients whilst $u$ is the field variable. The $\Omega$ is the computational domain, $\partial \Omega$ is the domain boundary and $\boldsymbol{n}$ is the outward unit normal vector on $\partial \Omega$. The second equation is a generalization of a Neumann boundary conditions, whereas the third equation is a general constraint with a Dirichlet boundary condition as a special case.

Comparing it with equation (1) the PDE coefficients and field variable can be written as,

$$
\begin{gathered}
k=\left(\begin{array}{cc}
\lambda+\sigma \alpha^{2} T & \sigma \alpha T \\
\sigma \alpha & \sigma
\end{array}\right) f=\left(\begin{array}{c}
\sigma\left((\vec{\nabla} V)^{2}+\sigma \vec{\nabla} T \vec{\nabla} V\right) \\
\vec{\nabla}\left([\varepsilon] \vec{\nabla} \frac{\partial V}{\partial t}\right)
\end{array}\right) \quad d_{a}=\left(\begin{array}{c}
\rho c \\
0
\end{array}\right) \\
u=\left(\begin{array}{c}
T \\
V
\end{array}\right)
\end{gathered}
$$

whilst the other coefficients of equation (2) are zero.

We used the Dirichlet boundary conditions to establish one electrode grounded and free evolution of temperature, while the other electrode is fixed at room temperature and a square voltage is applied to it. Running this simulation will be equivalent to vacuum conditions, as no convective terms for the films are considered. A simple parametric sweep can be used to simulate different thicknesses but in order to approach to the 2D structures it would be interesting to change the area of the film at least 100 times bigger than its thickness.

We added a box of air to simulate the effects of convection and conduction from the thermoelectric material to the air. For that purpose, we used the conjugate heat transfer module of COMSOL®. Then, we considered the heat equation in fluid for the air box, whose continuity and momentum equations are

$$
\begin{gathered}
\frac{\partial \rho}{\partial t}+\nabla(\rho v)=0 \\
\rho \frac{\partial v}{\partial t}+\rho(v \cdot \nabla) v=\nabla\left[-p \mathbf{I}+\mu\left(\nabla v+(\nabla v)^{T}\right)-\frac{2}{3} \mu(\nabla \cdot v) \mathbf{I}\right]+\mathbf{F}
\end{gathered}
$$

where $v$ is the velocity field, $\rho$ and $\mathrm{C}$ are the density and the heat capacity at constant pressure, $p$ is the pressure, $\mu$ is the dynamic viscosity and $\mathbf{I}$ is the identity matrix. Finally, the body force vector, $\mathrm{F}=-\mathrm{g}$. $\rho(T)$, must be considered to simulate the effects of gravity in convection. The temperature at the sides of the box of air was fixed at room temperature whilst the rest evolved freely.

Regarding the simulation of section 3.1.2., we introduce a new PDE equation for the electrodes and wire equivalent to the previous one of the appendix (1). Dirichlet boundary conditions were used to fix the top part of the wire at room temperature and the film with the electrodes and the wires was embedded in a box of air of $3 \times 3 \mathrm{~mm}$ big to simulate effects of natural convection. Parametric sweeps were used to simulate different electrical conductivity of the electrodes and different radius of the wire.

In all simulations, we considered a fine mesh for the structure until no changes on the results of the simulation were observed with the increase of the number of elements. 


\section{References}

[1]. Dresselhaus, M.S., et al., New Directions for Low-Dimensional Thermoelectric Materials. Advanced Materials, 2007. 19(8): p. 1043-1053.

[2]. Hicks, L.D. and M.S. Dresselhaus, Effect of quantum-well structures on the thermoelectric figure of merit. Physical Review B, 1993. 47(19): p. 12727-12731.

[3]. Bottner, H., et al., New thermoelectric components using microsystem technologies. Microelectromechanical Systems, Journal of, 2004. 13(3): p. 414-420.

[4]. Span, G., et al., Miniaturized TEG with thermal generation of free carriers. physica status solidi (RRL) - Rapid Research Letters, 2007. 1(6): p. 241-243.

[5]. Harman, T.C. and Harman, Special techniques for measurements of thermoelectric properties. Journal of applied physics, 1958. 29(9): p. 1373-1374.

[6]. Harman, T.C. and Harman, Multiple stage thermoelectric generation of power. Journal of applied physics, 1958. 29(10): p. 1471-1473.

[7]. Iwasaki, H. and H. Hori, Thermoelectric property measurements by the improved Harman method, ICT: 2005 24th International Conference on Thermoelectrics. 24th International Conference on Thermoelectrics, 2005: p. 501-504.

[8]. Iwasaki, H., M. Koyano, and H. Hori, Evaluation of the figure of merit on thermoelectric materials by harman method. Japanese journal of applied physics, 2002. 41(Part 1, No. 11A): p. 66066609.

[9]. Iwasaki, H., et al., Evaluation of the figure of merit of thermoelectric modules by Harman method. Japanese journal of applied physics, 2003. 42(Part 1, No. 6A): p. 3707-3708.

[10]. Fujimoto, S., et al., Development of transient measurement method for investigating thermoelectric properties in high temperature region. Japanese journal of applied physics, 2006. 45(11): p. 8805-8809.

[11]. Venkatasubramanian, R., et al., Thin-film thermoelectric devices with high room-temperature figures of merit. Nature, 2001. 413(6856): p. 597-602.

[12]. Castillo, E., C. Hapenciuc, and T. Borca Tasciuc, Thermoelectric characterization by transient Harman method under nonideal contact and boundary conditions. Review of scientific instruments, 2010. 81(4): p. 044902.

[13]. Singh, R., et al., Direct measurement of thin-film thermoelectric figure of merit. Applied physics letters, 2009. 94(21): p. 212508.

[14]. Singh, R., et al., Transient harman measurement of the cross-plane ZT of InGaAs/InGaAlAs superlattices with embedded ErAs nanoparticles. Materials Research society symposium proceedings, 2006. 886: p. 123-128.

[15]. Antonova, E.E. and D.C. Looman, Finite elements for thermoelectric device analysis in ANSYS, ICT 2005. 24th International Conference on Thermoelectrics, 2005: p. 215-203.

[16]. Martín-González, M., Caballero-Calero, O., and Diaz-Chao, P., Nanoengineering thermoelectrics for 21st century: Energy harvesting and other trends in the field. Renewable and Sustainable Energy Reviews, 2013. 24(0): p. 288-305.

[17]. Muñoz-Rojo, M., Caballero Calero, O., Lopeandia, A. F., Rodriguez-Viejo, .J., and Martin-Gonzalez M., Review in measurement techniques of transport properties of nanowires. Nanoscale, 2013.

[18]. Seifert, W., M. Ueltzen, and E. Muller, One-dimensional modelling of thermoelectric cooling. Physica status solidi. A, Applied research, 2002. 194(1): p. 277-290.

[19]. Vicente-Manzano, C., Rojas, A., Decepida, M., Abad, B., Feliz, Y., Caballero-Calero, O., Borca Tasciuc, D.A., Martín-Gonzalez, M., et al., Thermoelectric properties of Bi2Te3 films by constant and pulsed electrodeposition. Journal of Solid State Electrochemistry, 2013. 17(7): p. 2071-2078. 
[20]. Kasap, S., Thermoelectric Effects in Metals: Thermocouples. Principles of Electronic Materials and Devices (Selected Topic). 2006: McGraw-Hill.

Figures captions:

FIG.1. Schematic view of the signals measured by the Harman method at high and low frequencies. The left side illustrates the measurement setup: a freestanding thermoelectric thin film connected to a voltage source and a voltmeter. a) In the low frequency regime, a Seebeck voltage raise/decay is observed when the applied current is changing. b) In the high frequency regimes, temperature gradients cannot be established and thus the Seebeck voltage component is negligible.

FIG.2. a) Steady-state temperature distribution in a $60 \mu \mathrm{m} \mathrm{Bi}{ }_{2} \mathrm{Te}_{3}$ under a $10 \mathrm{mV}$ pulse of 0.05 s length. b) Temperature and voltage as function of time for the applied voltage pulse. Only the response to the first half of the pulse is shown.

FIG.3.Upper bound of low frequency, $f_{L F}$, and lower bound of high frequency, $f_{H F}$, as a function of film thickness. b) $\mathrm{ZT}$ and maximum difference of temperature $(\Delta \mathrm{T})$ obtained from the simulation.

FIG.4. Comparison of the lower bound of the high frequency predicted by analytical model and by simulation data. The model is in good agreement with the simulated data.

FIG.5. $f_{H F}$ as function of power factor and thermal conductivity for a $60 \mu \mathrm{m}$ p-type $\mathrm{Bi}_{2} \mathrm{Te}_{3}$ sample. The power factor was modified keeping constant the electrical conductivity and varying $\mathrm{S}$, and vice-versa resulting in the same results.

FIG.6. $f_{H F}$ as a function of the thickness of materials with different thermal conductivities and same power factor. The dots represent the simulated values while the lines are calculations using Eq. 5.

FIG.7 $f_{H F}$ and $f_{L F}$ dependence versus NW radius. The dashed line shows the typical electronic devices limitation when measuring frequency. Schematic view of the NW sample with electrodes painted in brown is shown in the inset of the graph.

FIG.8. a) The Temperature difference (right) and the total electrical voltage (left) generated at steady- state across a 60 um thick p-type $\mathrm{Bi}_{2} \mathrm{Te}_{3}$ as a function of the electrode electrical conductivity and for an applied voltage of $10 \mathrm{mV}$. Trend lines are used as a guide to the eye. b) The lower bound of the high frequency regime and extrinsic ZT as a function of the electrical conductivity of the electrodes.

FIG.9. a) Temperature distribution for a system composed of a $60 \mu \mathrm{m}-\mathrm{Bi}_{2} \mathrm{Te}_{3}$ film with a copper wire connected on top. b) $f_{H F}$ and temperature difference $(\Delta \mathrm{T})$ and c) total voltage and extrinsic ZT obtained as a function of the copper wire radius.

FIG.10. $f_{H F}$ as a function of the length of the wire. The dots represent the simulated values while the lines are calculations using Eq. 7.

FIG.11. Theoretical and simulated $Z T$ values and high frequencies $\left(\mathrm{f}_{\mathrm{HF}}\right)$ determined in different conditions. 


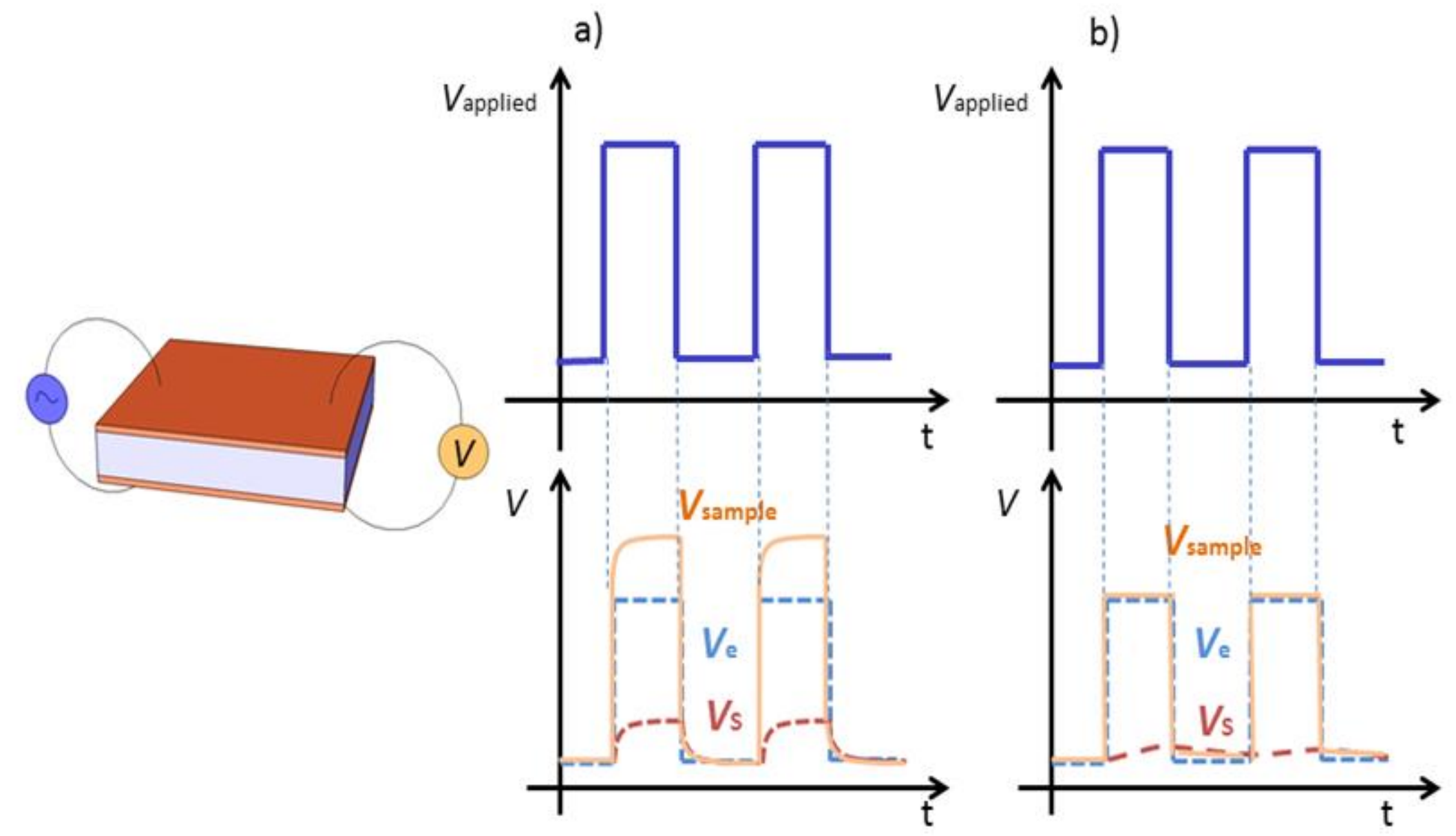


a)

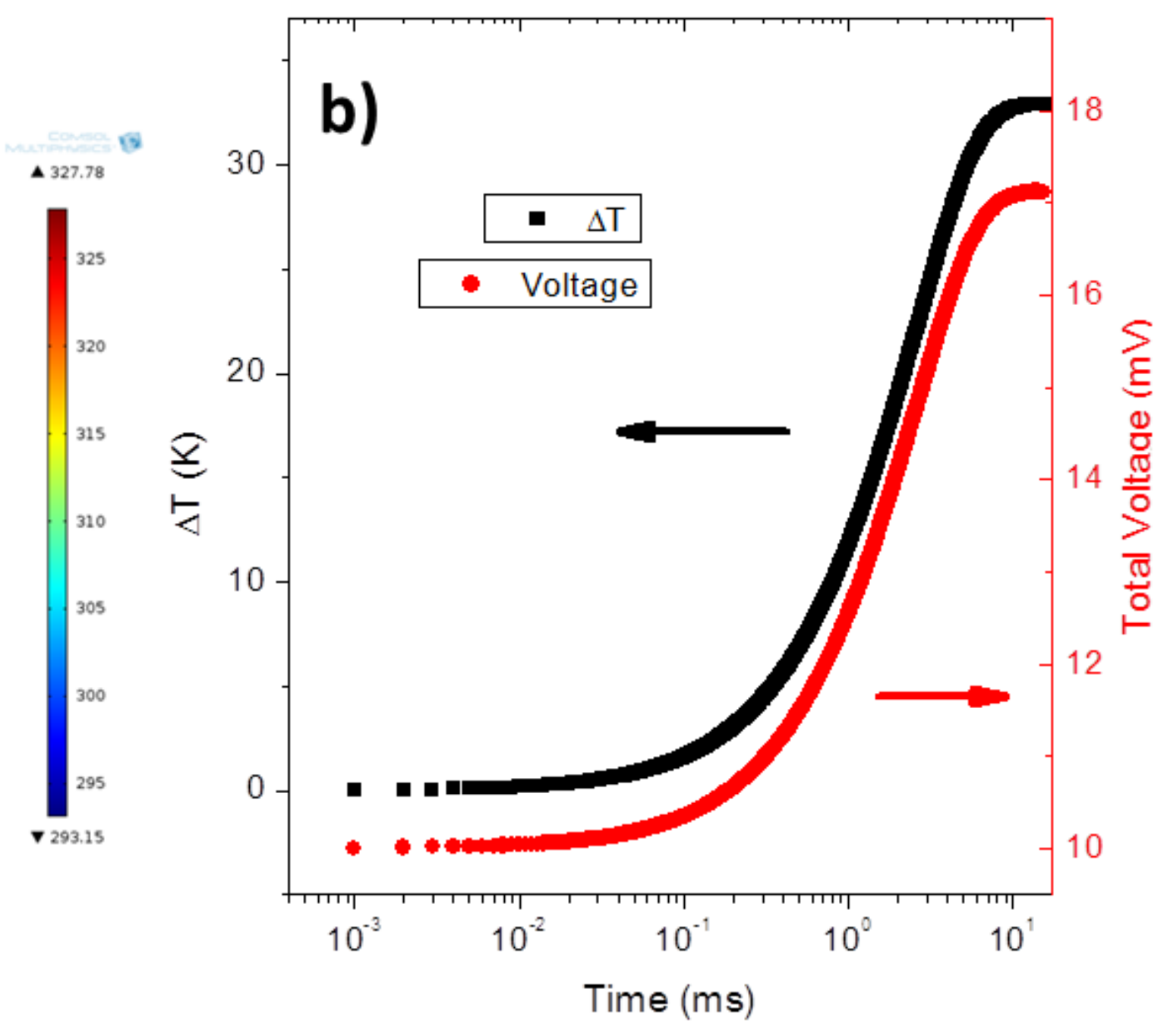


a)

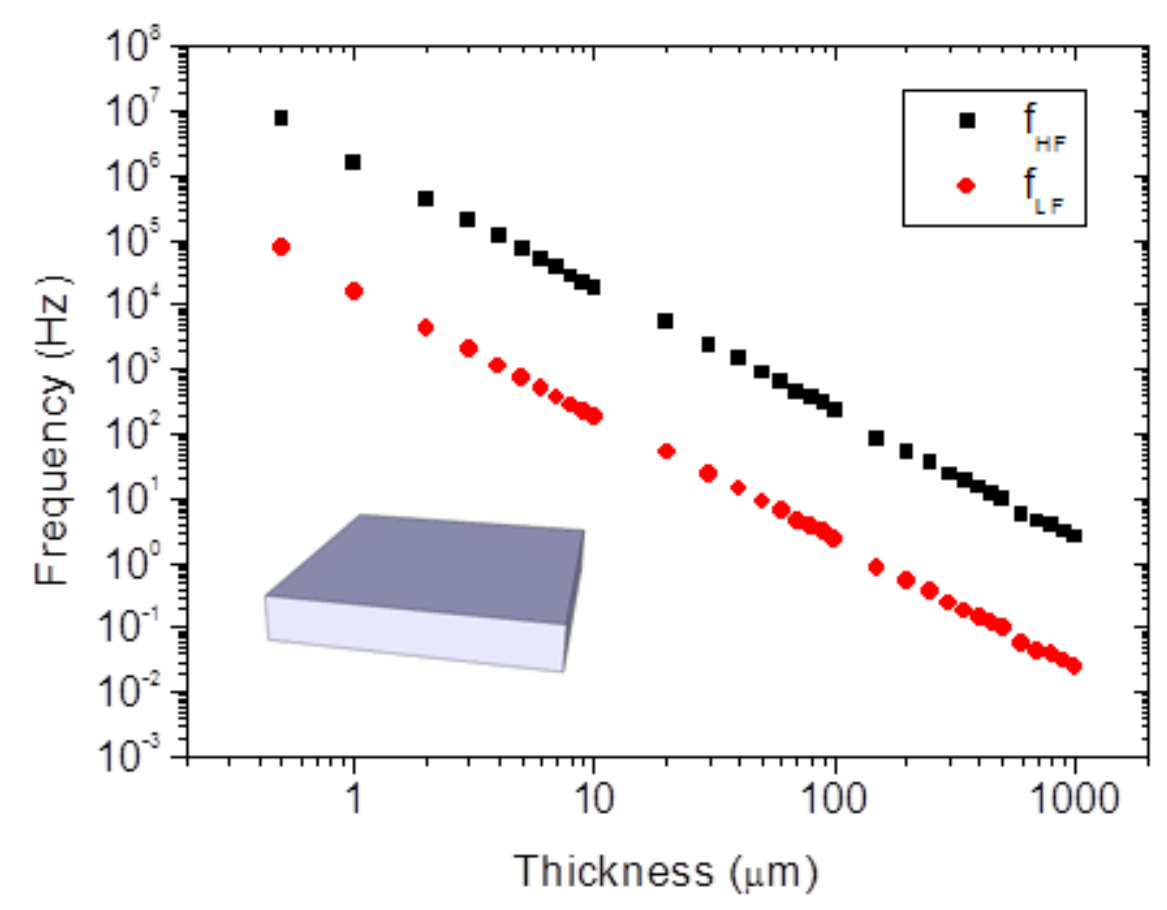

b)

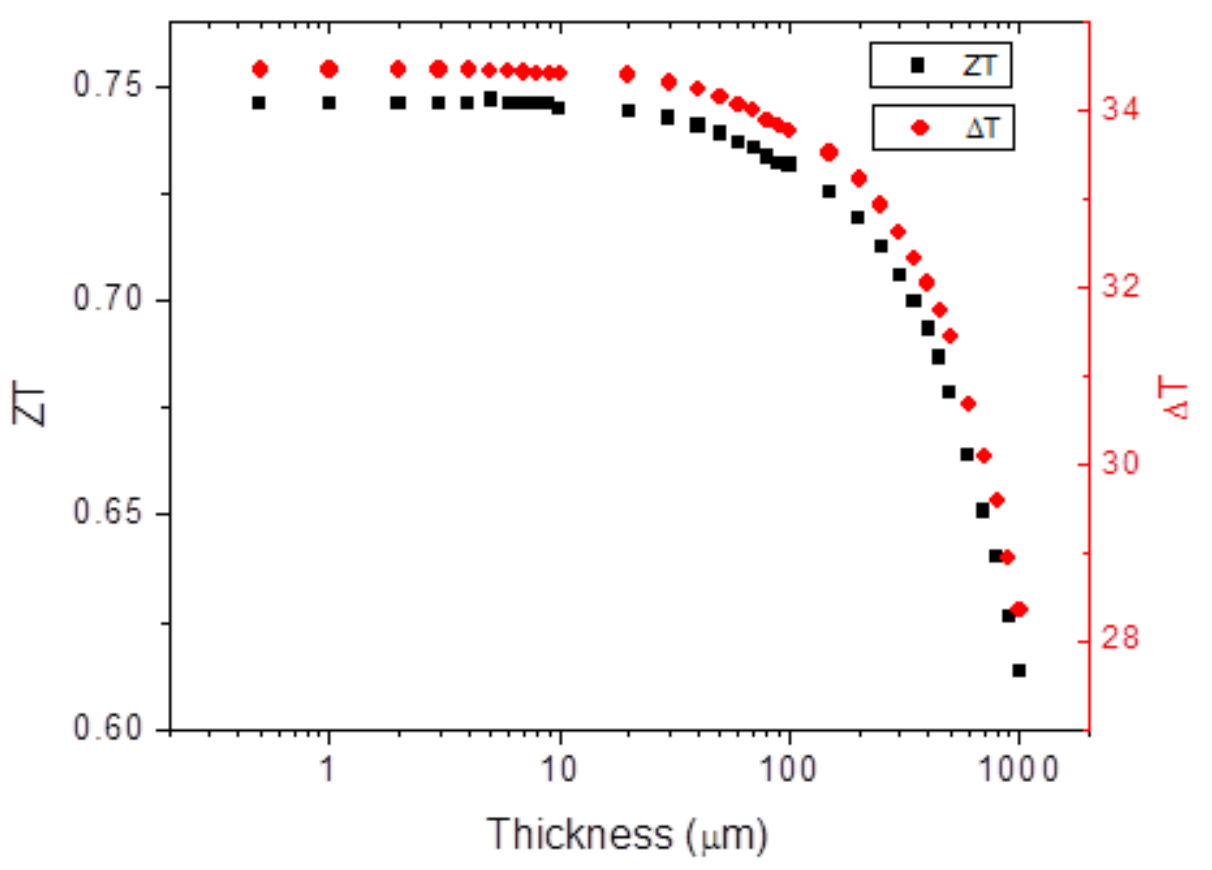




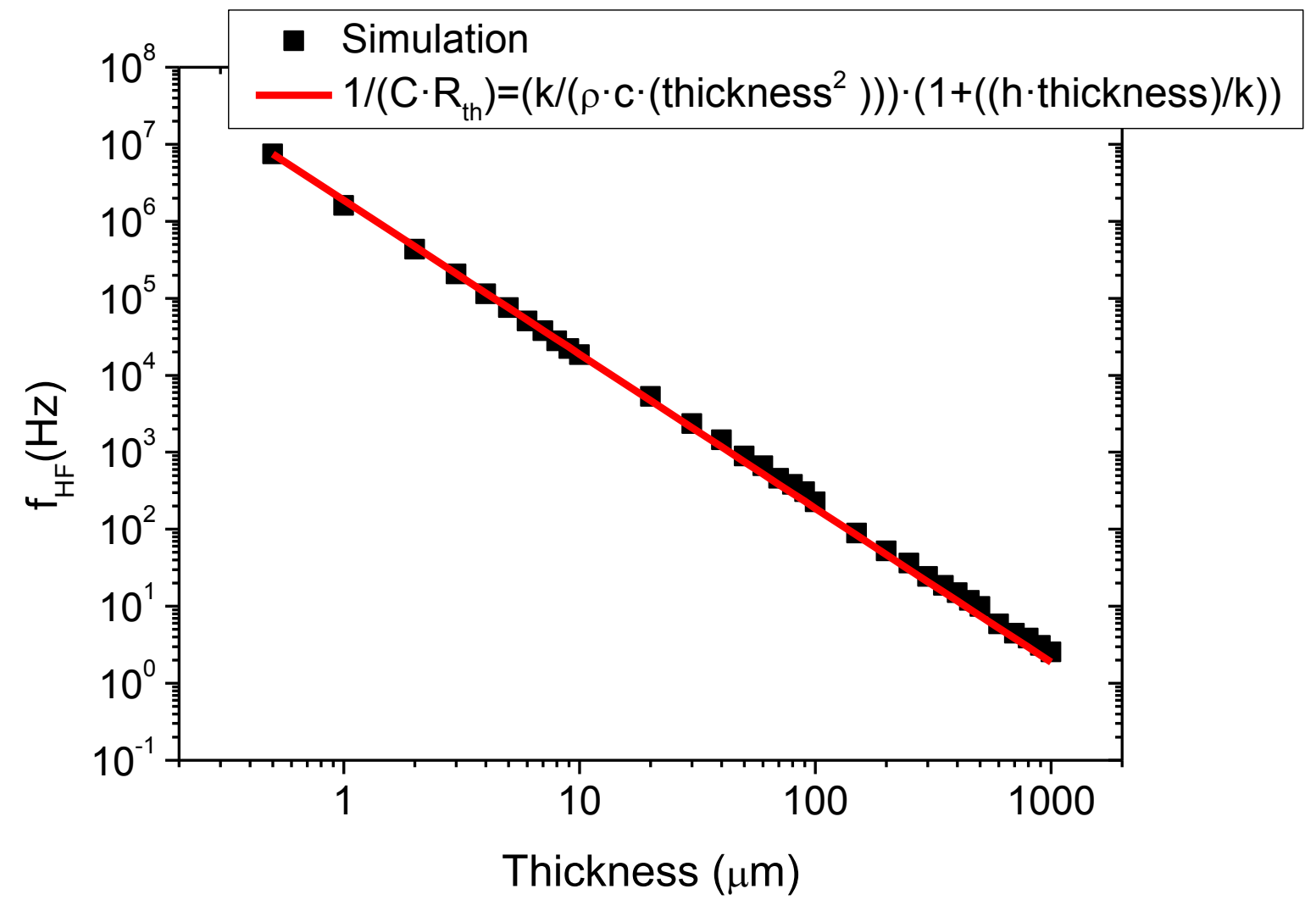




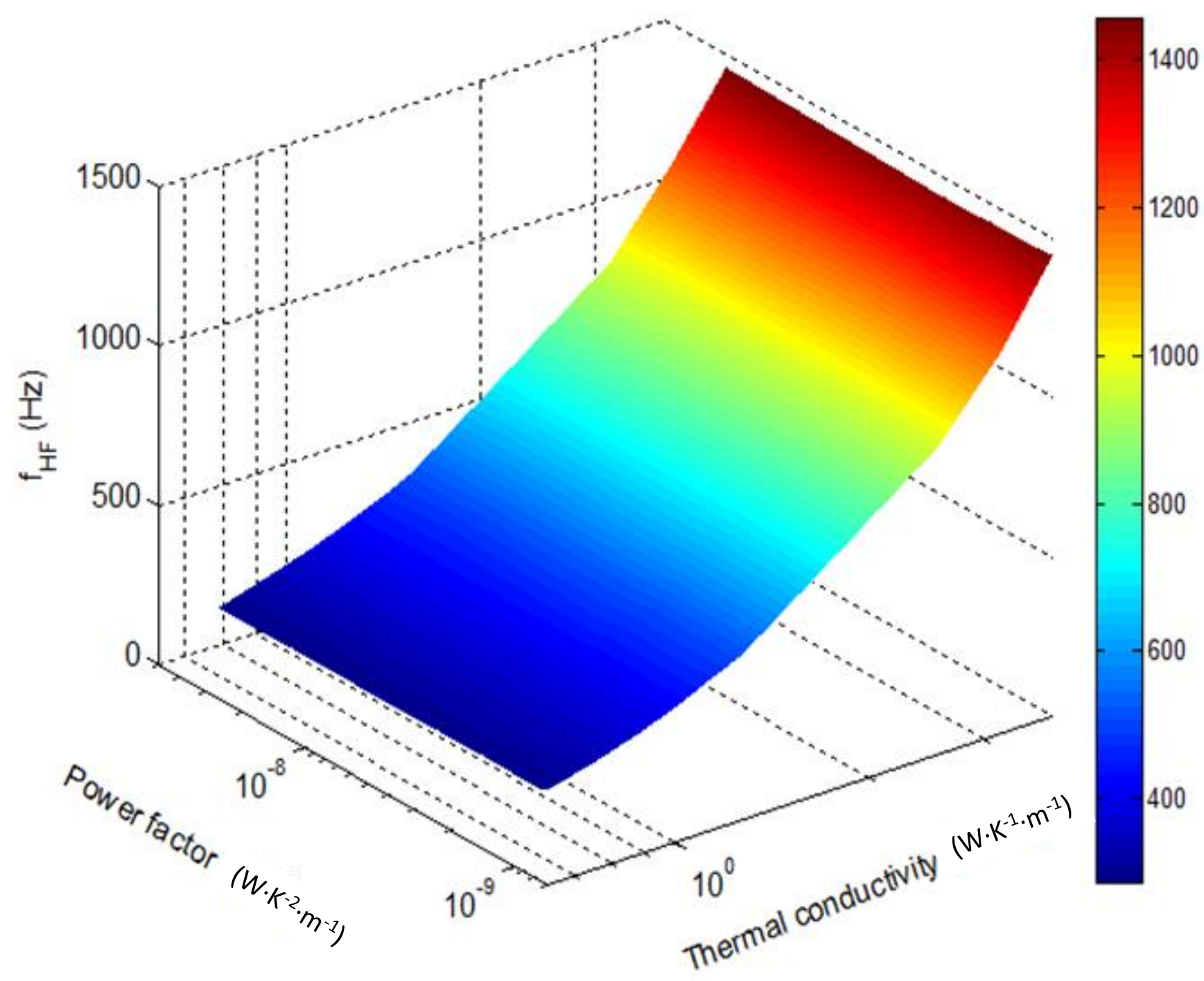




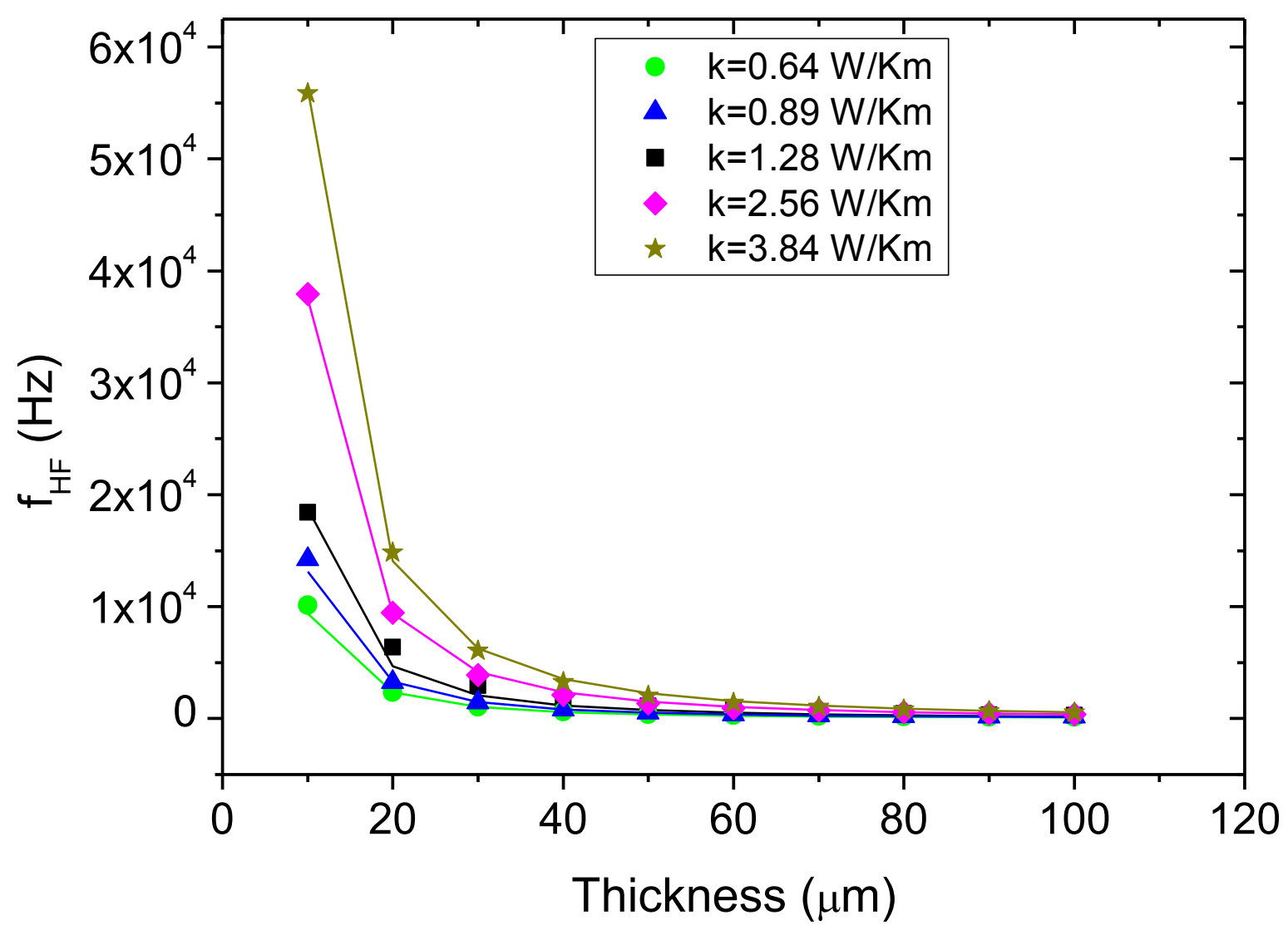




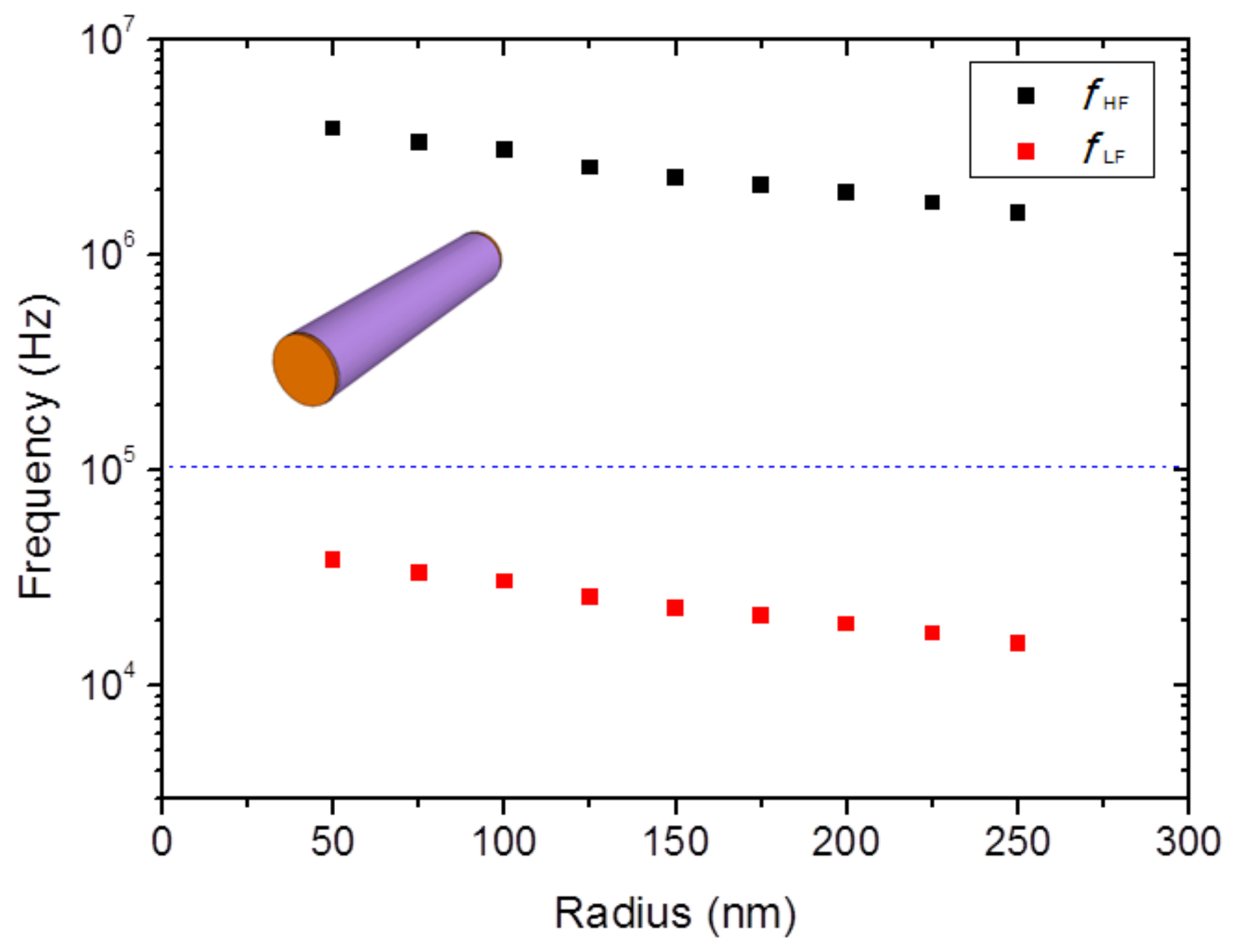


a)

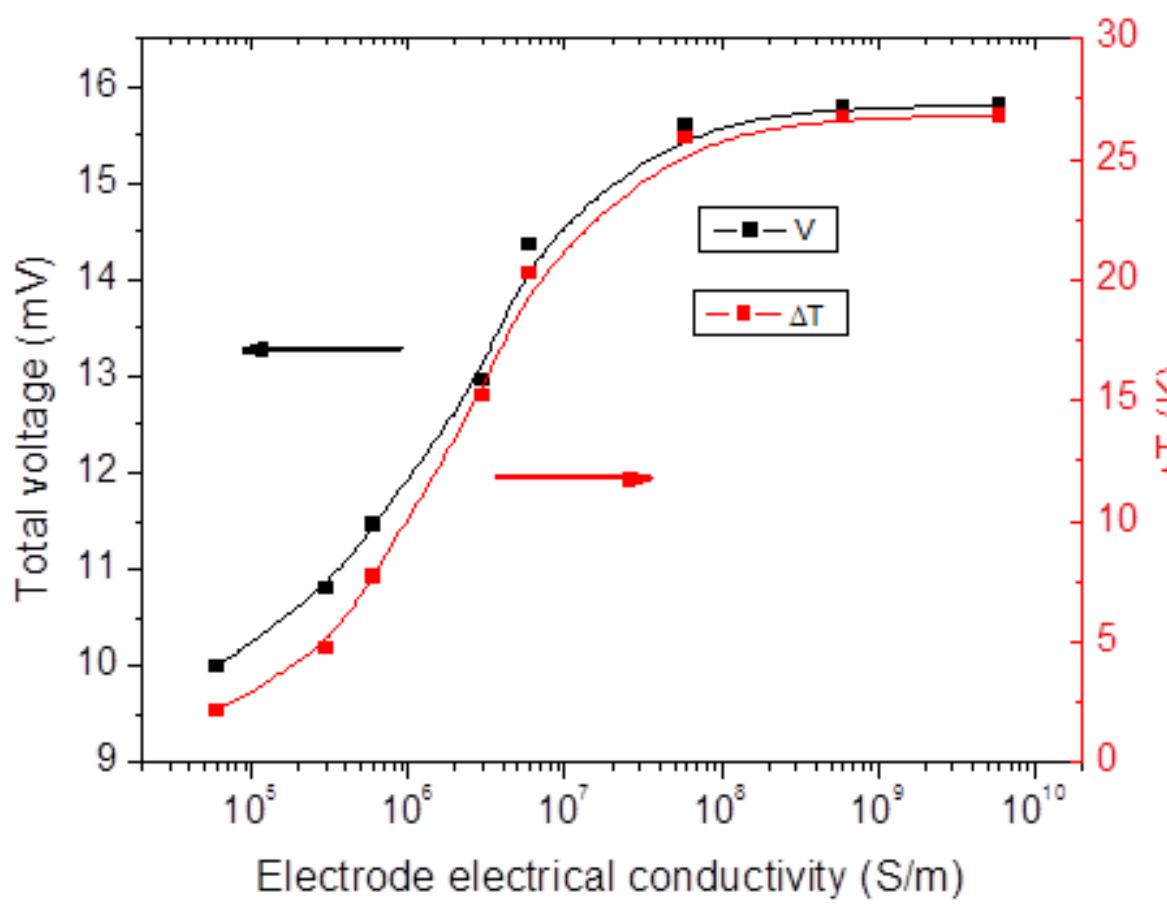

b)

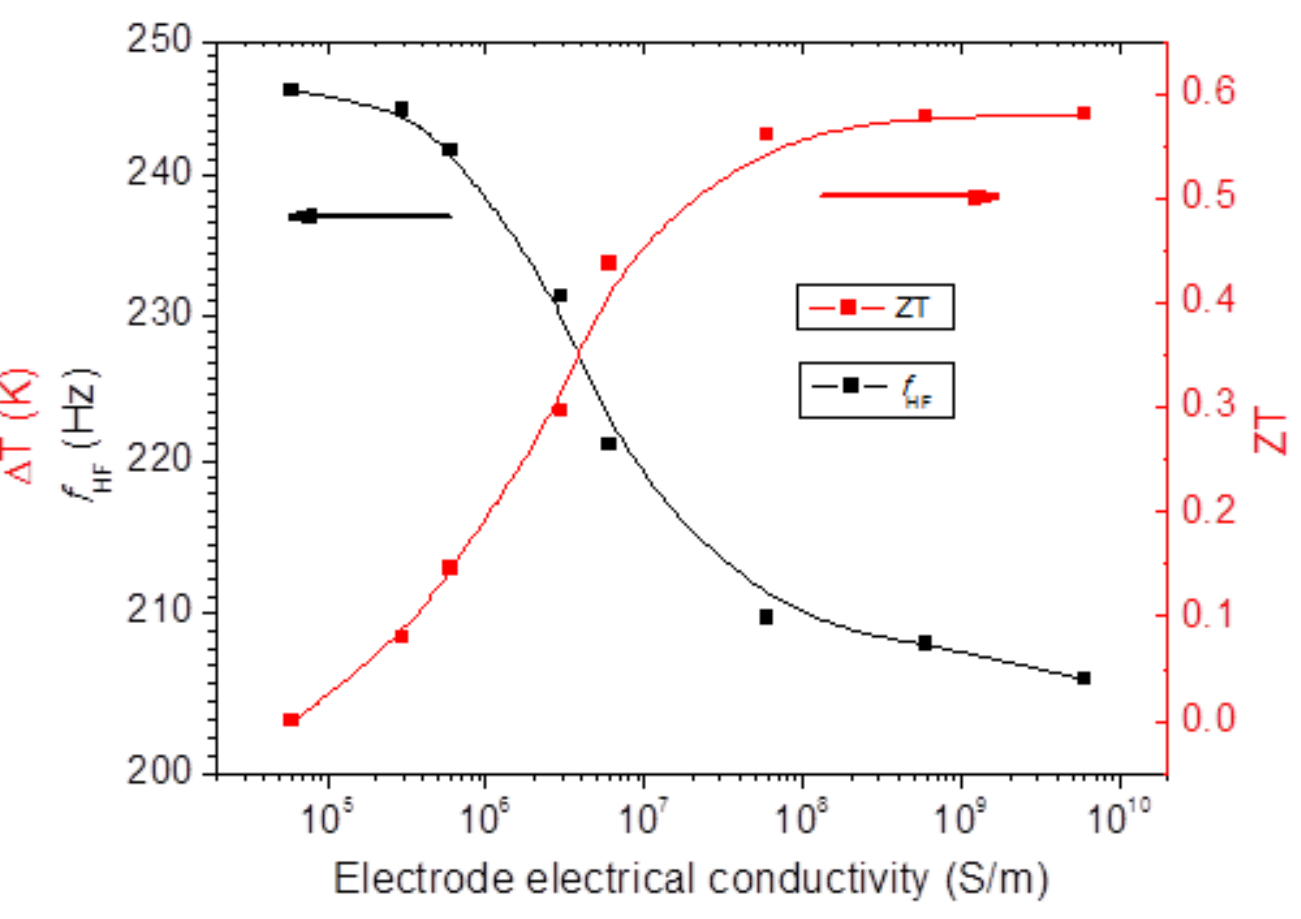



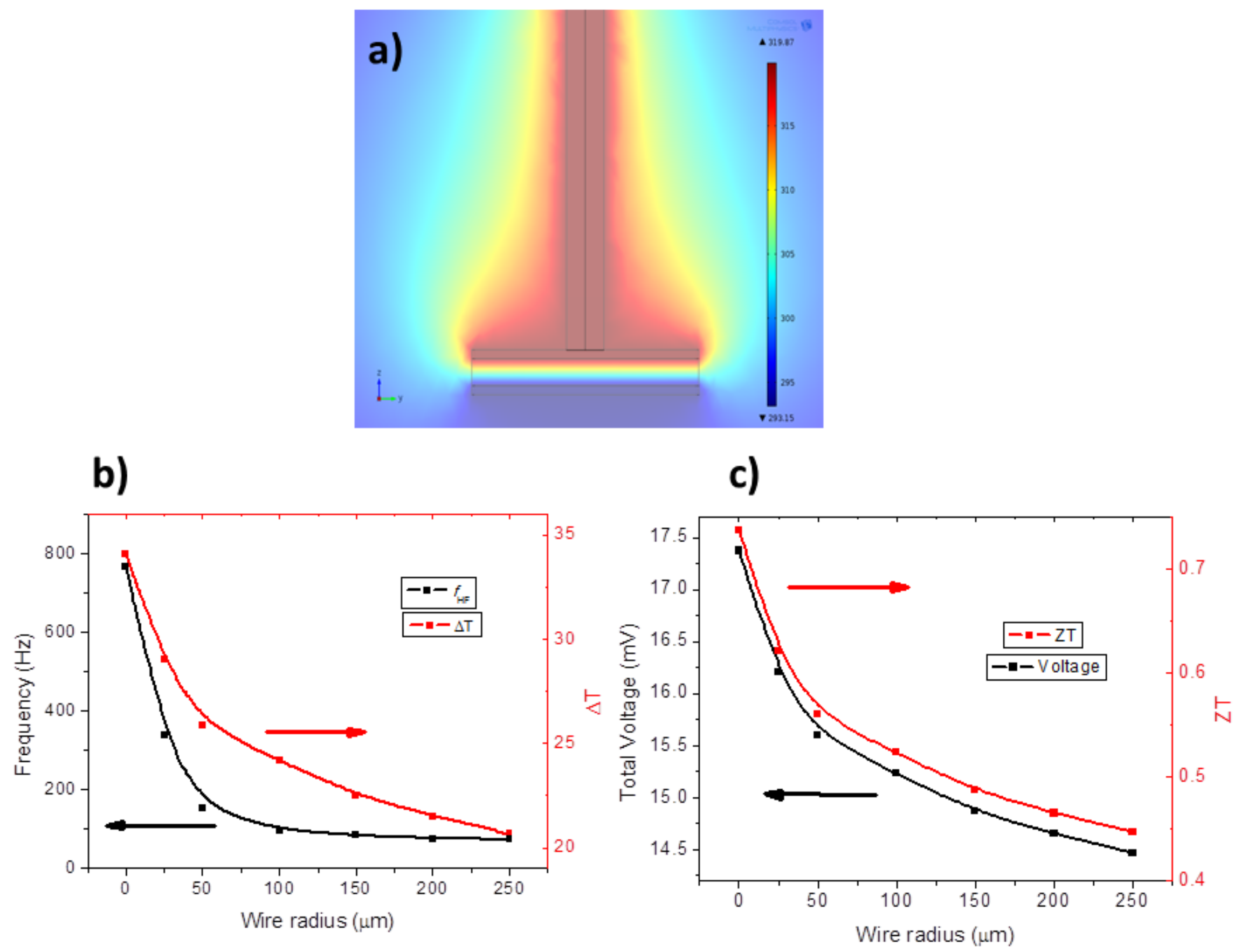


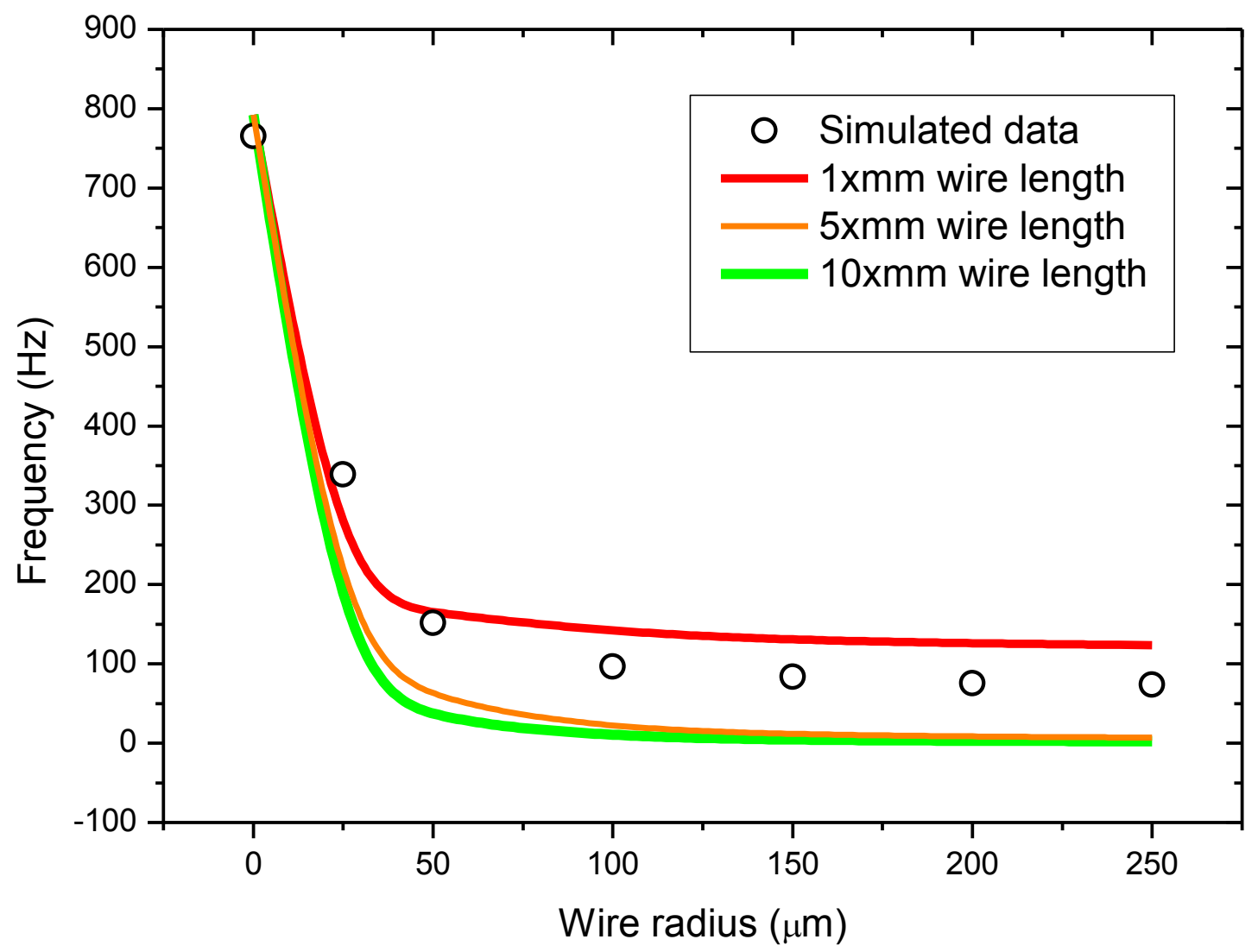




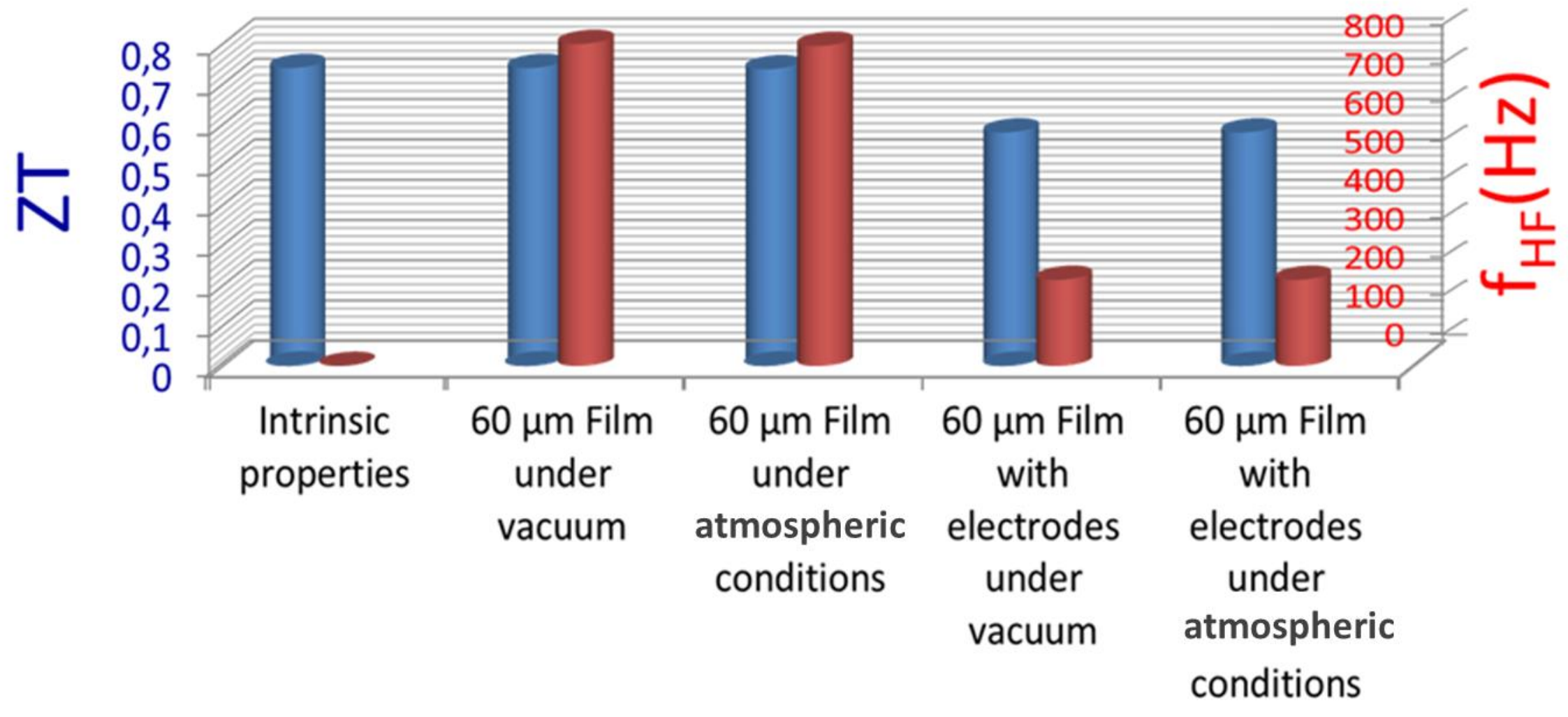

